\title{
BMJ Open Barriers and facilitators for implementation of electronic consultations (eConsult) to enhance specialist access to care: a scoping review protocol
}

\author{
Mohamed A Osman, ${ }^{1}$ Kara Schick-Makaroff, ${ }^{2}$ Stephanie Thompson, ${ }^{1}$ \\ Robin Featherstone, ${ }^{3,4}$ Liza Bialy, ${ }^{3,4}$ Julia Kurzawa, ${ }^{1}$ Ikechi G Okpechi, ${ }^{5}$ \\ Syed Habib, ${ }^{1}$ Soroush Shojai, ${ }^{1}$ Kailash Jindal, ${ }^{1}$ Scott Klarenbach, ${ }^{1}$ Aminu K Bello ${ }^{1}$
}

To cite: Osman MA, SchickMakaroff K, Thompson S, et al. Barriers and facilitators for implementation of electronic consultations (eConsult) to enhance specialist access to care: a scoping review protocol. BMJ Open 2018;8:e022733. doi:10.1136/ bmjopen-2018-022733

- Prepublication history and additional material for this paper are available online. To view these files, please visit the journal online (http://dx.doi.org/ 10.1136/ bmjopen-2018-022733).

Received 7 March 2018 Revised 19 May 2018 Accepted 15 June 2018

Check for updates

(C) Author(s) (or their employer(s)) 2018. Re-use permitted under CC BY-NC. No commercial re-use. See rights and permissions. Published by BMJ.

For numbered affiliations see end of article.

Correspondence to

Dr Aminu K Bello;

aminu1@ualberta.ca

\section{ABSTRACT}

Introduction Electronic consultations (eConsult), asynchronous exchanges of patient health information at a distance, are increasingly used as an option to facilitate patient care and collaboration between primary care providers and specialists. Although eConsult has demonstrated success in increasing efficiency in the referral process and enhancing access to care, little is known about the factors influencing its wider adoption and implementation by end users. In this paper, we describe a protocol to conduct a scoping review of the literature on the barriers and facilitators to a wider adoption and implementation of eConsult service.

Methods and analysis This scoping review will be based on the framework pioneered by Arksey and 0'Malley and later developed by Levac et al. We will use the guidance for scoping reviews developed by the Joanna Briggs Institute to report our findings. In addition to several electronic databases (Medline, Embase, Cochrane Library, CINAHL, EBSCOhost and PsycINFO) studies will be identified by including relevant grey literature. Two reviewers will independently screen titles and full texts for inclusion. Studies reporting on barriers and/or facilitators in settings similar to eConsult will be included. Data on study characteristics and key barriers and facilitators will be extracted. Data will be analysed thematically and classified using the Quadruple Aim framework.

Ethics and dissemination Approval by research ethics board is not required since the review will only include published and publicly accessible data. Review findings will be used to inform future studies and the development of practice tools to support the wider adoption and success of eConsult implementation. We plan to publish our findings in a peer-reviewed journal and develop a useful and accessible summary of the results.

\section{INTRODUCTION}

In many healthcare systems, particularly in Canada, ${ }^{1} \mathrm{UK}^{2}$ and in managed care models in the USA, ${ }^{3}$ primary care providers (PCPs) are the first point of contact for patients,

\section{Strengths and limitations of this study}

- To the best of our knowledge, this scoping review will be the first comprehensive examination of the literature on factors that favour or hinder the adoption of electronic consultations (eConsult) in patient care.

- The review is based on consultation with methodological experts and consultation with the target audience, thus facilitating translation of findings into useful tools for eConsult implementations.

- The study will leverage scoping review methodology; some known limitations of this methodology include absence of quality appraisal and risk of bias assessment for included studies; however, we plan to report on study designs and the use of statistical analyses.

and specialists rely on them to appropriately refer patients for specialist care. The demand for specialist care is growing and wait times to consult a specialist remain long. ${ }^{45}$ In the recent Commonwealth survey, Canada had the longest wait time to see a specialist $(>4$ weeks) among 11 developed countries who participated in the survey. ${ }^{6}$ Further, despite the increased adoption and uptake of electronic medical records (EMRs) in the country by PCPs (Alberta 87\%, national $75 \%$ ), ${ }^{6-8}$ most of the referrals and consultations to specialists are still done outside of the EMRs, such as through paper, fax and/or 'curbside consultations' (ie, in person, by telephone or email). These alternative methods of consultation present many challenges: telephone calls and in-person consultations require simultaneous presence of both providers and are often undocumented; emails may risk patients privacy; while paper and fax referrals 


\begin{tabular}{|c|c|}
\hline Domain & Definition \\
\hline \multicolumn{2}{|l|}{ A. Synchronous domains } \\
\hline Video-conferencing & $\begin{array}{l}\text { Use of real-time video and } \\
\text { audio for communicating } \\
\text { (consulting, teaching, } \\
\text { discussing treatment). }\end{array}$ \\
\hline $\begin{array}{l}\text { Phone (Mobile Health; } \\
\text { mHealth) }\end{array}$ & Telephone call. \\
\hline $\begin{array}{l}\text { Continuous remote } \\
\text { monitoring }\end{array}$ & $\begin{array}{l}\text { Real-time continuous feed } \\
\text { of information at a distance } \\
\text { (wearable biosensors, ECG). }\end{array}$ \\
\hline \multicolumn{2}{|l|}{ B. Asynchronous domains } \\
\hline Store-and-forward & $\begin{array}{l}\text { Transmission via email of } \\
\text { medical or laboratory data } \\
\text { and images to an expert for } \\
\text { remote review. }\end{array}$ \\
\hline Phone (mHealth) & $\begin{array}{l}\text { Use of SMS or other } \\
\text { methods of mobile } \\
\text { text messaging for } \\
\text { communication. }\end{array}$ \\
\hline $\begin{array}{l}\text { Self-monitoring and } \\
\text { management }\end{array}$ & $\begin{array}{l}\text { Involves one or more types } \\
\text { of sensors deployed in, on } \\
\text { or around a human body to } \\
\text { collect physiological signals. }\end{array}$ \\
\hline
\end{tabular}

Adapted with permission from Osman et al. ${ }^{18}$

Abbreviations: SMS, short message service.

are fragmented and may be lost, resulting in delays and duplications. ${ }^{9}$ Overall, evidence identifies that the referral process from primary to secondary care is suboptimal, and that many challenges and opportunities for improvement in efficiency remain. ${ }^{10}{ }^{11}$ In Canada, national survey results have demonstrated dissatisfaction with the current process of referral by patients, ${ }^{12}$ PCPs and specialists. ${ }^{11}$

Health information technologies in the form of telemedicine hold the potential to bridge the gap between primary and specialist care. ${ }^{13}$ Electronic consultations (eConsult) are being adopted in many countries to streamline the pathway of communication between different providers and reduce inefficiencies. ${ }^{15} 16$ Telemedicine is an all-inclusive term that includes any exchange of health information at a distance to provide and support healthcare using information technology. ${ }^{17}$ Telemedicine has different modalities that include both real-time (synchronous) or store-and-forward (asynchronous) exchange of information (table 1 ) ${ }^{18}$ eConsult is an asynchronous form of telemedicine that involves the transfer of health information between PCPs and specialists through a secure environment (shared electronic health record or web-based platform).${ }^{19} 20$ Typically, these systems are in the format of a standardised electronic form whereby a PCP would ask a patient-specific question and the referring specialist would either respond to the question, request more information or schedule a face-to-face consultation. ${ }^{19}$ Benefits of eConsult include enhanced communication between providers as PCPs and specialists are able to track their communications, review patient data and share personal information over a secure network. It also leads to increase overall referral efficiency by decreasing the need for face-to-face consultation while increasing access to specialist care. ${ }^{1621}$

Although eConsult is not yet widely adopted across Canada, the Ontario-based multispecialty Champlain BASE eConsult has pioneered its implementation and showed benefits in reduced wait times, avoidance of inappropriate referrals to specialists and potential cost savings to patients and the health system. ${ }^{21-23}$ In Alberta, researchers within the Interdisciplinary Chronic Disease Collaboration in collaboration with Alberta Health Services launched and piloted the eConsult service specific for kidney care, leveraging the province-wide secure shared electronic health records system (Netcare). ${ }^{24}$ With the eConsult system, PCPs are able to either submit a request for a face-to-face specialist consultation or request specialist advice. Evaluation of this pilot is ongoing. As eConsult systems are early in adoption, remuneration varies across jurisdictions; however, in the above described examples, providers and specialists can bill for these consultations. ${ }^{25}$

To our knowledge, this is the first scoping review aimed at identifying barriers and facilitators to eConsult adoption. In a qualitative study on the opinion of decision-makers in 16 different organisations that adopted eConsult systems in the USA, Tuot et al identified a number of facilitators including engaged leadership, early adopters, provider incentives, user-friendly technology and integration with EMRs, as well as barriers including provider resistance, lack of reimbursement, liability concerns and lack of integration into EMRs. ${ }^{26}$ This scoping review will build on this preliminary review, widening the geographical scope and using a rigorous framework. ${ }^{26}$

The literature demonstrates clear benefits of using eConsult, however, it is not widely adopted and little is known about the barriers and facilitators affecting wider implementation and adoption by key stakeholders. This information would allow for expansion of eConsult services across specialties and jurisdictions and could improve the effectiveness of existing systems. We, therefore, aim to undertake a broad examination of the eConsult implementation literature, including published and grey literature, focusing on barriers and facilitators to eConsult implementation. The key objective is to identify the factors reported by key stakeholders (patients, policy-makers and PCPs) that affect implementation of an eConsult to inform strategies for implementation and wider uptake.

\section{METHODS AND ANALYSIS}

\section{Theoretical basis}

When designing and implementing a new health system delivery tool like eConsult, it is important to identify the factors that favour and hinder its wider uptake by the 
Table 2 A comparison of scoping and systematic reviews

\begin{tabular}{|c|c|}
\hline Scoping review & Systematic review \\
\hline Broad research question. & Focused research question. \\
\hline $\begin{array}{l}\text { No critical appraisal of } \\
\text { included studies. }\end{array}$ & $\begin{array}{l}\text { Quality and risk of bias } \\
\text { assessment included. }\end{array}$ \\
\hline $\begin{array}{l}\text { Research protocol developed } \\
\text { but it involves iterative } \\
\text { approach with changes based } \\
\text { on initial search results. }\end{array}$ & $\begin{array}{l}\text { Research protocol developed } \\
\text { a priori. }\end{array}$ \\
\hline $\begin{array}{l}\text { More qualitative than } \\
\text { quantitative synthesis. }\end{array}$ & Often quantitative ana \\
\hline $\begin{array}{l}\text { Used in 'mapping the } \\
\text { literature' to identify gaps in a } \\
\text { body of literature, identify key } \\
\text { terms and concepts. }\end{array}$ & $\begin{array}{l}\text { Used to formulate a } \\
\text { conclusion about a focused } \\
\text { research question; assesses } \\
\text { the quality of existing } \\
\text { evidence. }\end{array}$ \\
\hline
\end{tabular}

Adapted with permission from Brien et al. ${ }^{31}$

end users (eg, PCPs). ${ }^{27}$ However, there is little evidence about these factors and how they influence eConsult adoption. ${ }^{26}$ Given that this is a complex intervention, it is challenging to study these factors using conventional systematic review methodology. However, a scoping review provides the means to map key concepts and gaps in evidence through a comprehensive review of the literature. ${ }^{28}$ It is useful in 'mapping' the evidence, especially in an emerging field like eConsult. ${ }^{29}$ One advantage of scoping reviews over systematic reviews is that scoping reviews typically incorporate different study designs and qualities which can be grouped to evaluate a particular topic of interest (table 2) ${ }^{3031}$ Furthermore, the breadth of content that is obtained through a scoping review allows for a high-level overview of all interventions/contexts in the literature and therefore, the potential to identify gaps in knowledge that are more suitable for in-depth and narrow focus study-such as in systematic reviews. We chose this method because we are anticipating that the search on eConsult implementation will yield a low number of heterogeneous study designs that may not be suitable for a systematic review.

\section{Approach}

To guide our scoping review protocol, we leveraged the framework first described by Arksey and O'Malley ${ }^{32}$ and later developed by Levac et a $\hat{P}^{33}$ (online supplementary appendix 1), which includes five steps: (1) identifying the research question; (2) identifying the relevant studies; (3) study selection; (4) charting the data and (5) reporting the results; (6) Consultation exercise-optional step. To report our findings, we will use the guidance on reporting scoping reviews recently published by the Joanna Briggs Institute (figure 1). ${ }^{30}$

Identifying the research question

The underlining question for this scoping review to answer is 'What is known about the barriers to and facilitators for the wider implementation of eConsult to enhance access to specialist care?'. Keeping with our broader objective of this review, any determinants in the eConsult literature that can influence implementation positively or negatively will be synthesised as barriers and facilitators at patient, provider and healthcare system levels. We used preidentified themes ${ }^{24}$ and published literature ${ }^{26}$ on barriers and facilitators to guide identifying these determinants.

\section{Search strategy and terms}

We will identify studies by conducting comprehensive searches of the following bibliographic databases: Ovid Medline (1946-), Ovid Embase (1988-), Wiley Cochrane Library (inception-), CINAHL via EBSCOhost (1937-) and Ovid PsycINFO (1987-). Our search will use both index (subject headings) and text words, and will combine concepts for eConsult, primary care and specialist care. In addition to these electronic databases, we will also search ProQuest Dissertations \& Theses Global, and we will include relevant grey literature by searching Conference Proceedings Citation Index (Clarivate Analytics) and screening the first five pages ( $\mathrm{n}=100$ results in total) from a Google search. Finally, through citation chaining (backward by one step) we will review the reference lists of included studies for relevant studies not identified from our initial search until saturation is achieved (ie, when there are no new studies being identified). The specific search strategies (for the selected databases and other data sources) will be developed and executed by an experienced information specialist (RF) and peer reviewed by a second medical librarian. No language or date restrictions will be applied to the search strategy (online supplementary appendix 2). Search results will be exported to EndNote V.X7 (Clarivate Analytics) and duplicates will be removed before the file is provided to reviewers for screening and data extraction.

\section{Selection criteria}

The following criteria will be used by the reviewers (table 3):

\section{Inclusion criteria}

1. Human studies.

2. Studies reporting on barriers and facilitators in storeand-forward (asynchronous) telemedicine settings similar to eConsult reported by patients, PCPs and specialists.

\section{Exclusion criteria}

1. Studies reporting on barriers and facilitators in real-time (synchronous) telemedicine settings, such as video-conferencing and continuous remote monitoring (table 1). We excluded these models of care because they are different from eConsult as described in the protocol here. They require both providers to be present at the same time and have different implementation barriers, such as the set-up which is more complex and often expensive. ${ }^{34}$

2. Image-based eConsult (ie, image transfer only for referral or consultation process, such as in telederma- 
Joanna Briggs Institute guidance
eConsult scoping review

Title: Barriers and facilitators for implementation of electronic consultations (eConsult) to enhance specialist access to care: a scoping review protocol

Background information on the process of care between PCPs and specialists, current state of eConsult research, and definition of scoping review methodology

Question: What is known about the barriers to and facilitators for the wider implementation of electronic consultations to enhance access to specialist care?

1) Human studies. 2) Studies reporting on barriers and facilitators in store and forward (asynchronous) telemedicine settings similar to eConsult

Patient, Primary care providers (PCPs) and specialists

The intervention of interest: eConsult as defined by asynchronous exchange of medical information between primary care and specialist remotely. The outcome measure: Perceived barriers and facilitators to eConsult. Description of the interve
phenomena of interest
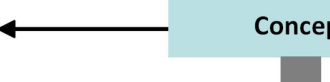

The clinical setting of interest is asynchronous telemedicine specifically electronic

consultations between PCPs and specialists.

Depending on the question of the study e.g. discipline.
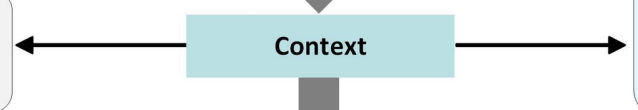

Two reviewers will independently screen all identified citations. Data will be extracted from identified studies using data extraction tool.

Reference list will be reviewed until saturation

is achieved. and index terms 2) Full search using al identified keywords 3) Search of the reference list of all included studies

Include data on: Total number of studies, decision flow chart, draft charting form. Presenting the data in tables, figures or narrative form

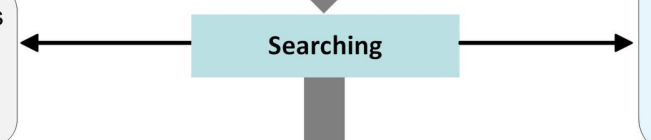

Data will be charted into tables and thematic synthesis based on the identified main themes in the literature of barriers and facilitators. Decision flow chart developed.

Potential limitations and mitigations strategies described as well as ethics and dissemination strategies.

The objective of the scoping review, rationale for developing the protocol and next research steps described.
Overall conclusion of the results; Future research directions based on gaps; Follow up (or not) with a systematic review and Recommendation for practice 


\begin{tabular}{|c|c|c|}
\hline & Inclusion criteria & Exclusion criteria \\
\hline Types of studies & $\begin{array}{l}\text { All study designs reporting on barriers and } \\
\text { facilitators to electronic consultations (eConsult) } \\
\text { will be included, such as randomised controlled } \\
\text { trial, qualitative, quantitative, mixed-methods } \\
\text { studies, reviews, dissertations and thesis. }\end{array}$ & - Study protocols. \\
\hline Types of interventions & $\begin{array}{l}\text { eConsultlike settings as defined by } \\
\text { asynchronous exchange of medical information } \\
\text { between primary care and specialist remotely. }\end{array}$ & $\begin{array}{l}\text { Studies reporting on barriers and facilitators in } \\
\text { other telemedicine settings (ie, synchronous } \\
\text { domains). } \\
\text { Studies reporting on only Image-based store- } \\
\text { and-forward (ie, teledermatology). } \\
\text { eConsult through non-secure portals (ie, email, } \\
\text { curbside consultations). }\end{array}$ \\
\hline
\end{tabular}

specialty that is uniquely different from other internal medicine specialties.

3. eConsult through non-secure portals (email, curbside consultations). Since security is central in the exchange of patients information electronically, we excluded these ways of communication as they may jeopardise patients privacy and are often informal and providers cannot be compensated for their work. ${ }^{36}$

\section{Data extraction}

The project lead (MAO) and another reviewer (LB) will independently screen all identified individual citations for potential inclusion. In the initial screening of title and abstracts, potentially relevant papers will be identified separately based on the inclusion and exclusion criteria, the two lists will be compiled, and full-text papers will be obtained. When agreement on a citation cannot be reached between the two reviewers, project supervisor (AKB) will be consulted for reconciliation.

Data will be extracted from eligible studies using a data extraction tool previously piloted on five papers (online supplementary appendix 3). Data items will include study characteristics, study design and key barriers and facilitators at patient, provider and health system levels. Data extraction will be conducted in three stages: (1) details of the paper, setting, population, methods, aims and conclusions will be listed; (2) each paper will be assessed for content in relation to predefined themes based on a preliminary review of the literature and the consultation exercise and (3) the key findings will be subjected to the predefined inclusion and exclusion criteria (table 3). All data will be extracted into an Excel file.

\section{Summarising and reporting the results}

In the analysis, following the recommendations to extend the scoping review process by adding thematic analysis, ${ }^{33}$ extracted data will be identified, analysed and interpreted qualitatively using both deductive (preidentified themes) and inductive (new identified themes) approaches. Using both approaches will allow us to develop our a priori themes further or expand them with new findings that did not fit in any of the previous categories. Textual data from included papers will be coded individually using a broad-based coding scheme by (MAO) and (LB) to look for common themes across papers. The analysis process will be augmented by using NVivo V.11 software.

The results will be summarised and organised into tables based on the evaluation framework of the study (Quadruple Aim) ${ }^{21} 37$ in forms of text (themes) and numbers (frequency). The tables will contain study authors, year of publication, methods (study design), reported barriers and facilitators for eConsult, and their frequency across the reported studies. We will also provide our search decision flowchart, as adopted from Preferred Reporting Items for Systematic Reviews and Meta-Analyses (PRISMA) guidelines (online supplementary appendix 4$).{ }^{38}$

We will use the Quadruple Aim framework to a guide our mapping of the literature on barriers and facilitators for eConsult. This framework for optimising health systems performance started as a Triple Aim for improving patient perspective and population's health while decreasing healthcare costs simultaneously ${ }^{39}$; recently a fourth dimension was added advocating for provider's perspective, making it a Quadruple Aim. ${ }^{37}$ This model has been proposed previously to evaluate eConsult. ${ }^{2136}$ In this scoping review, the provider's perspective is further subdivided into PCPs (referee) and specialist (referral recipient) perspectives; this division is helpful in this evaluation as the two providers encounter different set of factors influencing their use of health information technology. ${ }^{40}$ 
Table 4 Preidentified themes of potential eConsult barriers and facilitators ${ }^{24}$

\begin{tabular}{|c|c|}
\hline Facilitators & Barriers \\
\hline Improvement in care coordination & $\begin{array}{l}\text { Issues with privacy and } \\
\text { security }\end{array}$ \\
\hline Better clinical care & $\begin{array}{l}\text { Limited awareness and } \\
\text { ease of use }\end{array}$ \\
\hline $\begin{array}{l}\text { Disseminate best practice and } \\
\text { educational platform }\end{array}$ & $\begin{array}{l}\text { Aversion to adopt new } \\
\text { technology }\end{array}$ \\
\hline Facilitate better continuity of care & Required pace of change \\
\hline $\begin{array}{l}\text { Comprehensive data to make } \\
\text { decisions easily without the need } \\
\text { for a face-to-face consultation }\end{array}$ & Cost \\
\hline $\begin{array}{l}\text { Quick feedback to primary care } \\
\text { providers }\end{array}$ & Limited workforce \\
\hline $\begin{array}{l}\text { Clarity of information and } \\
\text { improved details }\end{array}$ & Lack of interest \\
\hline $\begin{array}{l}\text { Timeliness/reduced delays for } \\
\text { patient }\end{array}$ & Aversion to change \\
\hline $\begin{array}{l}\text { Convenience (eg, less travels for } \\
\text { patient) }\end{array}$ & Lack of time \\
\hline \multirow[t]{2}{*}{$\begin{array}{l}\text { Rapid triage and identification of } \\
\text { cases needing urgent care }\end{array}$} & $\begin{array}{l}\text { Convincing patients to } \\
\text { agree difficult }\end{array}$ \\
\hline & Compensation issues \\
\hline
\end{tabular}

\section{Quality assessment of included studies}

Quality appraisal and risk of bias assessment will not be conducted since it is not part of the scoping review methodology. ${ }^{30}{ }^{33}$ However, we will report on basic study characteristics, study design (observational vs RCT), the use of statistical and qualitative analysis and source of included studies (eg, peer-reviewed vs grey literature). Currently, a checklist for reporting is being developed by Tricco et al and will be added to PRISMA guidelines. ${ }^{41}$ Two independent reviewers will follow this a priori developed protocol at each step of the review (screening, data extraction). Included studies will be extracted into preidentified form (online supplementary appendix 3) and missing data from studies will be requested from the author team.

\section{Consultation exercise}

Arksey and O'Malley described a consultation process as an optional exercise for researchers conducting a scoping review. ${ }^{32}$ In the preimplementation phase of this study, we conducted a focus group of patients, policy-makers and $\mathrm{PCPs}^{24}$ in which we identified key themes of potential barriers and facilitators to eConsult service implementation specific to kidney care. We will use these preidentified themes (table 4) to assist in the analysis of this scoping review of the literature on barriers and facilitators of eConsult implementation across specialties.

\section{Patient and public involvement}

Patients and public were not involved at this stage of the project.

\section{ETHICS AND DISSEMINATION}

To disseminate our findings, we plan to publish our review and develop a useful and accessible summary of the results. Key deliverables of this review are (1) a scientific manuscript, (2) a one-page policy brief for decision-makers planning to adopt eConsult and (3) user-friendly summaries to the key stakeholders (patients, PCPs and specialists). Using an integrated knowledge translation approach, we involved potential intervention users from the study inception through our consultation exercise. Study findings will be used to engage leaders from Alberta Health Services and Primary Care Networks in Alberta and in the development of practice facilitation tools to support the wider adoption and success of eConsult implementation in the local context.

\section{LIMITATIONS AND ANTICIPATED PITFALLS}

The scoping review methodology has some known limitations. ${ }^{42} \mathrm{~A}$ common issue is the likelihood of not including a relevant study. To reduce the possibility of missing relevant publications, we plan to use several databases and search the reference lists of included studies for other potential studies until saturation is achieved. Another limitation is the absence of quality appraisal and risk of bias assessment of included studies. Since there is no quality assessment tool available for scoping reviews yet, we plan to report on the study characteristics and source of study (peer-reviewed vs grey literature). This review is built on previous work in which we preidentified potential barriers and facilitators from the literature. ${ }^{24}$ However, for practicality and to achieve the identified objectives, we will follow the rigorous yet feasible approach outlined in this protocol. We will also consult with an expert medical librarian (RF) and use the resources provided by The Alberta Strategy for Patient-Oriented Research (SPOR) SUPPORT Unit (https://sporresources.researchalberta.ca/).

We acknowledge that increase wait time to see specialist is multifactorial. For instance, in Canada many different elements (ie, workforce, finance, communication with providers) are contributing together to increase wait time and decrease access to specialist care. ${ }^{43}$ However, a report by the Wait Time Alliance identified structural changes in health service delivery as the way forward to address the issues of access to care. ${ }^{44}$ Such structural changes in process of care delivery can be seen in eConsult. It was demonstrated in different countries ${ }^{45} 46$ and among several specialties ${ }^{45} 46$ that using eConsult can increase access to specialist care and significantly impact wait times in a positive way.

In this scoping review, we will follow this protocol to guide our decision-making. Protocols are essential to guide the review process and have the potential to reduce changes during the review process, selective reporting and repetition of work. ${ }^{47}$ However, in the case of scoping reviews, most of the published work has no a priori protocol to follow (87\%) according to a report conducted by Tricco et $a t^{48}$ examining the methodology and reporting of scoping reviews in the literature. 


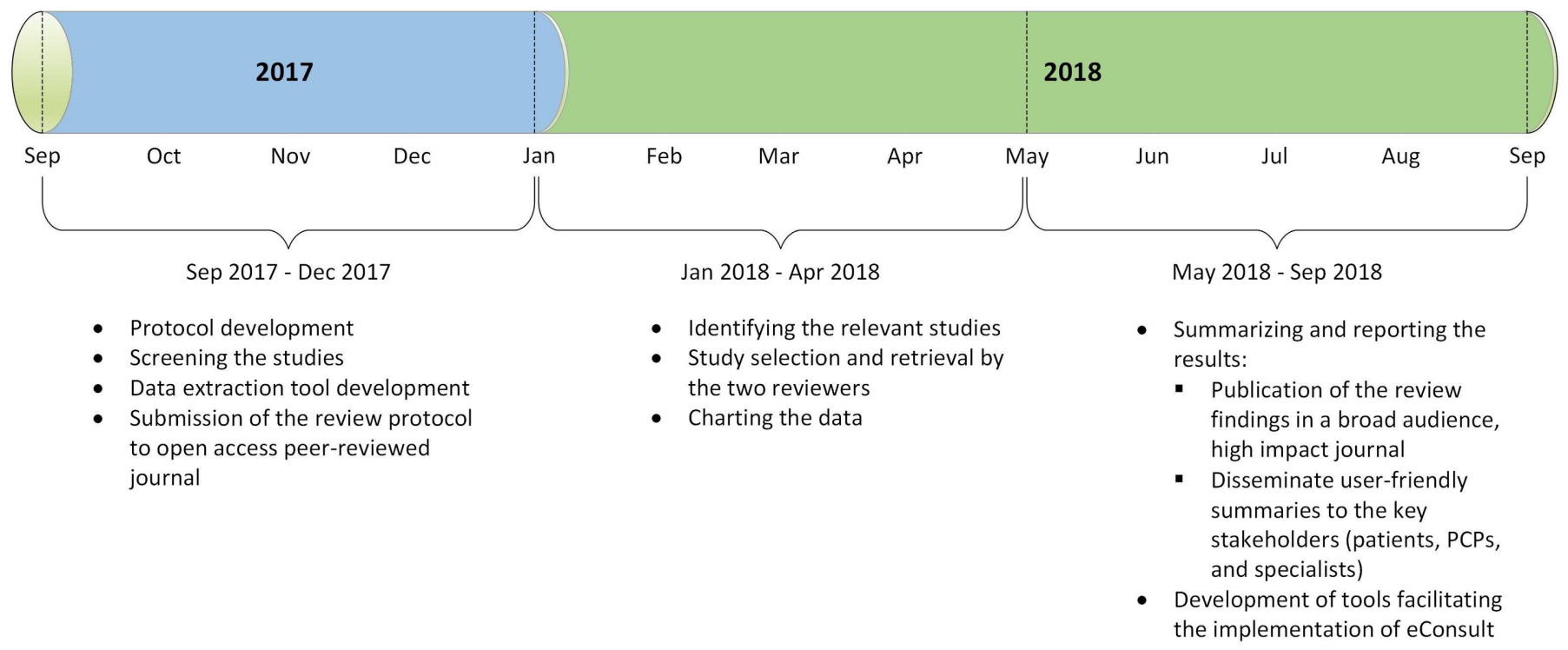

Figure 2 Proposed scoping review timeline. Abbreviations: eConsult, electronic consultations; PCPs, primary care providers.

\section{TIMELINE}

The proposed completion timeline for this study is 1 year. The timeline is divided into three main stages: stage 1: protocol development and conducting the search, stage 2: study selection and data extraction and stage 3: summarising and reporting the results (figure 2).

\section{DISCUSSION}

The findings of this planned scoping review will be used to define the current evidence on barriers and facilitators to wider adoption of eConsult. Moreover, it will be used to identify the current knowledge gaps in the literature, as well as the need for a systematic review for future research.

Assessing barriers and facilitators is an essential step in the implementation evaluation process. ${ }^{49}$ Although understanding the context ${ }^{50}$ on which eConsult was implemented is crucial to interpretation (local knowledge) ${ }^{49}$ identifying barriers and facilitators that influence implementation from the reported experiences in the literature (external knowledge) ${ }^{49}$ of similar initiatives is key to build on other's lessons and thus decrease the need to 'reinvent the wheel' every time. The findings of this review will be used to engage patients, PCPs and policy-makers in adopting eConsult in Alberta to enhance access to specialist care.

In the review, we plan to summarise factors influencing eConsult from both high volume early adopters as well as emerging users of eConsult, using different platforms (eg, web-based and shared electronic health record) and across different payment structures (eg, single payer and insurance mandate). Furthermore, synthesising these barriers and facilitators will help understand common variables to focus on how to modify barriers best and enhance facilitators to increase use and appeal of the intervention.

\section{CONCLUSION}

To our knowledge, this scoping review will be the first comprehensive examination of the literature on factors that influence the adoption of eConsult. The findings of the review will guide future studies and will be used to inform eConsult implementation and scale up in Canada and beyond.

\section{Author affiliations}

${ }^{1}$ Department of Medicine, University of Alberta, Edmonton, Alberta, Canada

${ }^{2}$ Faculty of Nursing, University of Alberta, Edmonton, Alberta, Canada

${ }^{3}$ Alberta SPOR SUPPORT Unit KT Platform, Edmonton, Alberta, Canada

${ }^{4}$ Alberta Research Centre for Health Evidence, University of Alberta, Edmonton, Alberta, Canada

${ }^{5}$ Division of Nephrology and Hypertension, University of Cape Town, Cape Town, South Africa

Acknowledgements Authors like to thank Marcello Tonelli, Brenda Hemmelgarn and Braden Manns for their contributions to the project. We thank Janice Kung for her help in the development of initial search strategy.

Contributors Following International Committee of Medical Journal Editors (ICMJE) authorship guidelines. AKB and MAO had the original idea for this study. MAO and AKB wrote the first draft of the manuscript. SK, ST, KS-M, JK, LB, IGO, SH, SS, KJ and RF contributed to the development of the idea, study protocol development and reviewed the manuscript for intellectual content. All authors approved the final submitted version of the manuscript.

Funding This work is funded by Northern Alberta Renal Program (NARP) and the Interdisciplinary Chronic Disease Collaboration (ICDC). The ICDC is funded through an Alberta Innovates Collaborative Research and Innovation Opportunity Team Grant.

Competing interests None declared.

Patient consent Not required.

Ethics approval Approval by research ethics board is not required since the review only involves published and publicly available data with no involvement of identifiable patient records.

Provenance and peer review Not commissioned; externally peer reviewed.

Open access This is an open access article distributed in accordance with the Creative Commons Attribution Non Commercial (CC BY-NC 4.0) license, which permits others to distribute, remix, adapt, build upon this work non-commercially, and license their derivative works on different terms, provided the original work is 
properly cited, appropriate credit is given, any changes made indicated, and the use is non-commercial. See: http://creativecommons.org/licenses/by-nc/4.0/.

\section{REFERENCES}

1. Hutchison B, Levesque JF, Strumpf E, J-f L, Erin CN, et al. Primary health care in Canada: systems in motion. Milbank $Q$ 2011;89:256-88.

2. Forrest CB. Primary care gatekeeping and referrals: effective filter or failed experiment? BMJ 2003;326:692-5.

3. Forrest CB, Nutting P, Werner JJ, et al. Managed health plan effects on the specialty referral process: results from the Ambulatory Sentinel Practice Network referral study. Med Care 2003;41:242-53.

4. Jaakkimainen L, Glazier R, Barnsley J, et al. Waiting to see the specialist: patient and provider characteristics of wait times from primary to specialty care. BMC Fam Pract 2014;15:16.

5. Barua B, Rovere M, Skinner BJ. Waiting your turn: Fraser Institute, 2015.

6. How Canada Compares: Results From The Commonwealth Fund 2015 International Health Policy Survey of Primary Care Physicians. 2016. https://www.cihi.ca/sites/default/files/document/ commonwealth_fund_2015_pdf_en.pdf (accessed 7 May 2018).

7. Collier R. National physician survey: EMR use at $75 \%$. CMAJ 2015;187:E17-E18.

8. Chang F, Gupta N. Progress in electronic medical record adoption in Canada. Can Fam Physician 2015;61:1076-84.

9. Cook DA, Sorensen KJ, Wilkinson JM. Value and process of curbside consultations in clinical practice: a grounded theory study. Mayo Clin Proc 2014;89:602-14.

10. Bichel A, Erfle S, Wiebe V, et al. Improving patient access to medical services: preventing the patient from being lost in translation. Healthc Q 2009;13 Spec No:61-8.

11. Candaian medical association. Experiences with Referrals: Results of two surveys. 2012. https://www.cma.ca/Assets/assets-library/ document/en/advocacy/28-Referrals-e.pdf (accessed 7 May 2018).

12. Liddy C, Poulin PA, Hunter Z, et al. Patient perspectives on wait times and the impact on their life: A waiting room survey in a chronic pain clinic. Scand J Pain 2017;17:53-7.

13. Ekeland AG, Bowes A, Flottorp S. Effectiveness of telemedicine: a systematic review of reviews. Int J Med Inform 2010;79:736-71.

14. Buntin MB, Burke MF, Hoaglin MC, et al. The benefits of health information technology: a review of the recent literature shows predominantly positive results. Health Aff 2011;30:464-71.

15. Stoves J, Connolly J, Cheung CK, et al. Electronic consultation as an alternative to hospital referral for patients with chronic kidney disease: a novel application for networked electronic health records to improve the accessibility and efficiency of healthcare. Qual Saf Health Care 2010;19:e54-e.

16. Straus $\mathrm{SG}$, Chen $\mathrm{AH}$, Yee $\mathrm{H}$, et al. Implementation of an electronic referral system for outpatient specialty care. AMIA Annu Symp Proc 2011;2011:1337.

17. Perednia DA, Allen A. Telemedicine technology and clinical applications. JAMA 1995;273:483-8.

18. Osman MA, Okel J, Okpechi IG, et al. Potential applications of telenephrology to enhance global kidney care. BMJ Glob Health 2017;2:e000292.

19. Vimalananda VG, Gupte G, Seraj SM, et al. Electronic consultations (e-consults) to improve access to specialty care: a systematic review and narrative synthesis. J Telemed Telecare 2015;21:323-30.

20. Liddy C, Maranger J, Afkham A, et al. Ten steps to establishing an e-consultation service to improve access to specialist care. Telemed J E Health 2013;19:982-90.

21. Liddy C, Drosinis P, Keely E. Electronic consultation systems: worldwide prevalence and their impact on patient care-a systematic review. Fam Pract 2016;33:274-85.

22. Liddy C, Afkham A, Drosinis $P$, et al. Impact of and satisfaction with a new econsult service: A mixed methods study of primary care providers. J Am Board Fam Med 2015;28:394-403.

23. Keely E, Li J, Magner P, et al. Nephrology econsults for primary care providers: Original investigation. Can J Kidney Health Dis 2018;5:205435811775361

24. Bello AK, Molzahn AE, Girard LP, et al. Patient and provider perspectives on the design and implementation of an electronic consultation system for kidney care delivery in Canada: a focus group study. BMJ Open 2017;7:e014784

25. Stanistreet K, Verma J, Kirvan K, et al. Physician remuneration for remote consults: An overview of approaches across Canada. Healthc Q 2017;20:12-15.

26. Tuot DS, Leeds K, Murphy EJ, et al. Facilitators and barriers to implementing electronic referral and/or consultation systems: a qualitative study of 16 health organizations. BMC Health Serv Res 2015;15:568.

27. Price M, Singer A, Kim J. Adopting electronic medical records: are they just electronic paper records? Can Fam Physician 2013;59:e322-e9.

28. Colquhoun HL, Levac D, O'Brien KK, et al. Scoping reviews: time for clarity in definition, methods, and reporting. J Clin Epidemiol 2014;67:1291-4.

29. Anderson S, Allen P, Peckham S, et al. Asking the right questions: scoping studies in the commissioning of research on the organisation and delivery of health services. Health Res Policy Syst 2008;6:7.

30. Peters MD, Godfrey CM, Khalil H, et al. Guidance for conducting systematic scoping reviews. Int J Evid Based Healthc 2015;13:141-6.

31. Brien SE, Lorenzetti DL, Lewis S, et al. Overview of a formal scoping review on health system report cards. Implement Sci 2010;5:2.

32. Arksey H, O'Malley L. Scoping studies: towards a methodological framework. Int J Soc Res Methodol 2005;8:19-32.

33. Levac D, Colquhoun H, O'Brien KK. Scoping studies: advancing the methodology. Implement Sci 2010;5:69.

34. Scott Kruse C, Karem P, Shifflett K, et al. Evaluating barriers to adopting telemedicine worldwide: A systematic review. J Telemed Telecare 2018;24:4-12.

35. Finnane A, Dallest K, Janda M, et al. Teledermatology for the diagnosis and management of skin cancer: a systematic review. JAMA Dermatol 2017;153:319-27.

36. Young MJ, Pham J. Improving the electronic nexus between generalists and specialists: A public health imperative? Healthc 2016;4:302-6.

37. Bodenheimer T, Sinsky C. From triple to quadruple aim: care of the patient requires care of the provider. Ann Fam Med 2014;12:573-6.

38. Moher D, Liberati A, Tetzlaff J, et al. Preferred reporting items for systematic reviews and meta-analyses: the PRISMA statement. PLoS Med 2009;6:e1000097.

39. Berwick DM, Nolan TW, Whittington J. The triple aim: care, health, and cost. Health Aff 2008;27:759-69.

40. Redd TK, Doberne JW, Lattin D, et al. Variability in electronic health record usage and perceptions among specialty vs. Primary care physicians. AMIA Annu Symp Proc 2015;2015.

41. Preferred Reporting Items for Systematic Reviews and MetaAnalysis:extension for Scoping Reviews (PRISMA-ScR) 2015. http:// www.equator-network.org/wp-content/uploads/2009/02/Executivesummary_ScR_Dec-9.pdf (accessed 7 May 2018).

42. Pham MT, Rajić A, Greig JD, et al. A scoping review of scoping reviews: advancing the approach and enhancing the consistency. Res Synth Methods 2014;5:371-85.

43. Vogel L. Canadians still waiting for timely access to care. CMAJ 2017;189:E375-E376.

44. 2014. Time to close the gap: report card on wait times in Canada http://www.waittimealliance.ca/wp-content/uploads/2014/06/FINALEN-WTA-Report-Card.pdf (accessed 7 May 2018).

45. Keely E, Liddy C, Afkham A, Utilization AA. Utilization, benefits, and impact of an e-consultation service across diverse specialties and primary care providers. Telemed J E Health 2013;19:733-8.

46. Barnett ML, Yee HF, Mehrotra A, et al. Los Angeles Safety-net program econsult system was rapidly adopted and decreased wait times to see specialists. Health Aff 2017;36:492-9.

47. Shamseer L, Moher D, Clarke M, et al. Preferred reporting items for systematic review and meta-analysis protocols (PRISMA-P) 2015 elaboration and explanation. BMJ 2015;349:g7647.

48. Tricco AC, Lillie E, Zarin W, et al. A scoping review on the conduct and reporting of scoping reviews. BMC Med Res Methodol 2016;16:15.

49. Graham ID, Logan J, Harrison MB, et al. Lost in knowledge translation: time for a map? J Contin Educ Health Prof 2006;26:13-24.

50. Moore GF, Audrey S, Barker M, et al. Process evaluation of complex interventions: Medical Research Council guidance. BMJ 2015;350:h1258. 